\title{
Stability analysis of linear positive systems with time delays on time scales
}

\author{
Chao Zhang ${ }^{*}$ and Yuangong Sun
}

\author{
* Correspondence: ss_zhangc@ujn. \\ edu.cn \\ School of Mathematical Sciences, \\ University of Jinan, Jinan, \\ Shandong 250022, P. R. China
}

\begin{abstract}
This article studies the necessary and sufficient conditions for asymptotic stability of the linear positive systems with bounded time-varying delays on time scales. Using Lyapunov functions, asymptotic stability conditions for positive systems with constant delays on time scales are established. The relations between the two types of systems have been found out, which bridged the gap between their stability. These results not only unify the existing ones of linear positive continuous- and discretesystems but also contain more complicated time scales.
\end{abstract}

Keywords: positive systems, delays, stability, time scales

\section{Introduction}

In the real world, many physical, biological and medical systems involve variables that have nonnegative sign, e.g., population levels, absolute temperature, and concentration of substances. Such systems are referred to as positive systems. A dynamical system is called positive if any trajectory of the system starting from non-negative initial states remains forever non-negative for non-negative controls. An overview of state of the art in positive systems theory is given in the monographs [1-3].

Stability is one of the most important properties of dynamic systems, and a massive monograph has been concentrated on this topic for positive systems [4-9]. In the last few years, conditions for stability and robust stability of linear positive discrete-time systems with delays have been given in [10-19].

Firstly, we introduce some notations. $\mathbb{N}\left(\mathbb{N}_{0}\right)$ denotes the set of positive (nonnegative) integers, $\mathbb{R}\left(\mathbb{R}_{0_{0,+}}\right)$ denotes the set of real (nonnegative) numbers, $\mathbb{R}^{n}\left(\mathbb{R}_{0,+}^{n}, \mathbb{R}_{+}^{n}\right)$ is $n$ dimensional linear vector space over real (nonnegative, positive) numbers with the maximum modulus norm $\|\cdot\|$ given by $\|x\|=\max _{i=1,2, \cdots, n}\left|x_{i}\right|, x \in \mathbb{R}^{n}$, and $\mathbb{R}^{n \times m}\left(\mathbb{R}_{0,+}^{n \times m}\right)$ is the set of all real (nonnegative) matrices of $(n \times m)$-dimension. For $A \in \mathbb{R}^{n \times m}$, we write $A \succcurlyeq 0(\preccurlyeq 0, \succ 0$, and $\prec 0)$ to indicate that all elements of matrix $A$ are nonnegative (non-positive, positive, and negative) and the notation of matrices will be used throughout this brief: $A=\left[a_{l g}\right]$ and $A_{i}=\left[a_{l g}^{(i)}\right]$.

In [9], Haddad and Chellaboina obtained a necessary and sufficient stability condition for the following continuous-time positive systems with constant delays:

$$
\begin{aligned}
& x^{\prime}(t)=A x(t)+A_{d} x(t-\tau), \quad t \geq 0 \\
& x(t)=\varphi(t), \quad t \in[-\tau, 0]
\end{aligned}
$$

(c) 2012 Zhang and Sun; licensee Springer. This is an Open Access article distributed under the terms of the Creative Commons Attribution License (http://creativecommons.org/licenses/by/2.0), which permits unrestricted use, distribution, and reproduction in any medium, provided the original work is properly cited. 
where $A_{d} \in \mathbb{R}_{0,+}^{n \times n}, a_{l g} \geq 0$ for $l \neq g, \tau \geq 0$, and $\varphi:[-\tau, 0] \rightarrow \mathbb{R}_{0,+}^{n}$ is the vector value initial function. They obtained the following result [9, Theorem 3.1]: the above continuous-time positive system is asymptotically stable if and only if there exists a vector $\lambda \in \mathbb{R}_{+}^{n}$ such that

$$
\left(A+A_{d}\right) \lambda \prec 0 .
$$

Recently, Liu et al. [19] employed a comparison result to establish some necessary and sufficient conditions for the following discrete-time positive systems with timevarying delays:

$$
\begin{aligned}
& x(t+1)=A_{0} x(t)+\sum_{i=1}^{p} A_{i} x\left(t-\tau_{i}(t)\right), \quad t \in \mathbb{N}_{0} \\
& x(t)=\varphi(t) \succcurlyeq 0, \quad t=-\tau, \ldots, 0,
\end{aligned}
$$

where $A_{i} \in \mathbb{R}_{0,+}^{n \times n}$ for $i \in\{0,1,2, \ldots, p\}, 0 \leq \tau_{i}(t) \leq \tau_{i}$ with $\tau_{i} \in \mathbb{N}, \tau=\max \left\{\tau_{i} \mid i=1,2, \ldots\right.$, $p\}$, and $\varphi:\{-\tau, \ldots, 0\} \rightarrow \mathbb{R}_{0,+}^{n}$ is the vector-valued initial function. They found out the following similar result [19, Theorem 1]: the above discrete-time positive system is asymptotically stable if and only if there exists a vector $\lambda \in \mathbb{R}_{+}^{n}$ such that

$$
\left(\sum_{i=0}^{p} A_{i}-I\right) \lambda \prec 0 .
$$

Since time scales is a tool for establishing a unified framework for continuous and discrete analysis. In the present article, we try to unify the necessary and sufficient conditions for asymptotic stability of linear positive continuous- and discrete-time systems with delays into one form on time scales.

This article is organized as follows. In Section 2, necessary preliminaries are presented, and some lemmas are provided. Section 3 proposes the necessary and sufficient stability criteria for positive systems with time-varying delays on time scales.

\section{Notations and preliminaries}

In this section, some basic definitions and some fundamental results are introduced, which are necessary for developing the main results of this article. Let $\mathbb{T}$ denote a time scales, that is, $\mathbb{T}$ is a nonempty closed subset of $\mathbb{R}$ Denote $\mathbb{T}_{0}=\{0\} \cup \mathbb{T}$ and $\mathbb{T}_{0,+}=\mathbb{R}_{0,+} \cap \mathbb{T}_{0}$. For simplicity, let $\underline{N}=\{1,2, \ldots, n\}, \underline{P}=\{1,2, \ldots, p\}$, and $\underline{P}_{0}=\{0\} \cup \underline{P}$, where $n$ and $p$ are positive integers.

Define the forward and backward jump operators $\sigma, \rho: \mathbb{T} \rightarrow \mathbb{T}$ by

$$
\sigma(t)=\inf \{s \in \mathbb{T}: s>t\} \text { and } \rho(t)=\sup \{s \in \mathbb{T}: s<t\},
$$

where $\inf \emptyset=\sup \mathbb{T}, \sup \emptyset=\inf \mathbb{T}$. A point $t \in \mathbb{T}$ is called right-scattered, right-dense, left-scattered, and left-dense if $\sigma(t)>t, \sigma(t)=t, \rho(t)<t$, and $\rho(t)=t$, respectively. We put $\mathbb{T}^{k}=\mathbb{T}$ if $\mathbb{T}$ is unbounded above and $\mathbb{T}^{k}=\mathbb{T} \backslash(\rho(\max \mathbb{T}), \max \mathbb{T}]$ otherwise. The graininess $\mu: \mathbb{T} \rightarrow[0, \infty)$ is defined by

$$
\mu(t)=\sigma(t)-t .
$$

Let $f$ be a function defined on $\mathbb{T} f$ is said to be (delta) differentiable at $t \in \mathbb{T}^{k}$ provided there exists a constant $a$ such that for any $\varepsilon>0$, there is a neighborhood $U$ of $t$ (i.e., 


$$
\begin{gathered}
|f(\sigma(t))-f(s)-a(\sigma(t)-s)| \leq \varepsilon|\sigma(t)-s|, \quad \forall s \in \mathcal{U} \text {. for some } \delta>0) \text { with } \\
|f(\sigma(t))-f(s)-a(\sigma(t)-s)| \leq \varepsilon|\sigma(t)-s|, \quad \forall s \in \mathcal{U} .
\end{gathered}
$$

In this case, denote $f^{A}(t):=a$. If $f$ is (delta) differentiable for every $t \in \mathbb{T}^{k}$, then $f$ is said to be (delta) differentiable on $\mathbb{T}$ If $f$ is differentiable at $t \in \mathbb{T}^{k}$, then

$$
f^{\Delta}(t)= \begin{cases}\lim _{s \rightarrow t s \in \mathrm{T}} \frac{f(t)-f(s)}{t-s}, & \text { if } \mu(t)=0 \\ \frac{f(\sigma(t))-f(t)}{\mu(t)}, & \text { if } \mu(t)>0 .\end{cases}
$$

If $F^{\Delta}(t)=f(t)$ for all $t \in \mathbb{T}^{k}$, then $F(t)$ is called an antiderivative of $f$ on $\mathbb{T}$. In this case, define the delta integral by

$$
\int_{s}^{t} f(\tau) \Delta \tau=F(t)-F(s) \quad \text { for all } s, t \in \mathbb{T} .
$$

Moreover, a function $f$ defined on $\mathbb{T}$ is said to be rd-continuous if it is continuous at every right-dense point in $\mathbb{T}$ and its left-sided limit exists at every left-dense point in $\mathbb{T}$.

Definition 1. A function $f: \mathbb{T} \rightarrow \mathbb{R}$ is said to be right-increasing at $t_{0} \in \mathbb{T} \backslash\{\max \mathbb{T}\}$ provided

(i) $f\left(\sigma\left(t_{0}\right)\right)>f\left(t_{0}\right)$ in the case that $t_{0}$ is right-scattered;

(ii) there is a neighborhood $U$ of $t_{0}$ such that $f(t)>f\left(t_{0}\right)$ for all $t \in U$ with $t>t_{0}$ in the case that $t_{0}$ is right-dense.

If the inequalities for $f$ are reverse in (i) and (ii), $f$ is said to be right-decreasing at $t_{0}$. The following result can be directly derived from (1).

Lemma 1. Assume that $f: \mathbb{T} \rightarrow \mathbb{R}$ is differentiable at $t_{0} \in \mathbb{T} \backslash\{\max \mathbb{T}\}$. If $f^{\wedge}\left(t_{0}\right)>0$, then $f$ is right-increasing at $t_{0}$; and if $f^{\wedge}\left(t_{0}\right)<0$, then $f$ is right-decreasing at $t_{0}$.

Consider the following delayed system

$$
\begin{aligned}
& x^{\Delta}(t)=\left(A_{0}-I\right) x(t)+\sum_{i=1}^{p} A_{i} x\left(t-\tau_{i}(t)\right), \quad t \in \mathbb{T}_{0,+} \\
& x(t)=\varphi(t) \succcurlyeq 0, \quad t \in[-\tau, 0] \cap \mathbb{T}_{0}
\end{aligned}
$$

where $A_{i} \in \mathbb{R}^{n \times n}$ for $i \in \underline{P}, \tau=\max _{i \in \underline{P}}\left\{\sup _{t \in \mathbb{T}_{0,+}} \tau_{i}(t)\right\}, t-\tau_{i}(t) \in \mathbb{T}_{0}, \tau_{i}(t) \in \mathbb{T}_{0,+}$ for $i \in \underline{P}, i \in \underline{P}$, and $\varphi:[-\tau, 0] \cap \mathbb{T}_{0} \rightarrow \mathbb{R}_{0,+}^{n}$ is the vector value initial function.

Definition 2. System (2) is said to be positive if and only if for any initial condition $\varphi(\cdot) \succcurlyeq 0$, the corresponding trajectory $x(t) \succcurlyeq 0$ holds for all $t \in \mathbb{T}_{0,+}$.

Lemma 2. If $A_{i} \succcurlyeq 0$ for all $i \in \underline{P}_{0}$ and $a_{j j}^{(0)} \geq 1$ for all $j \in \underline{N}$ then system (2) is positive.

Lemma 3. If system (2) is positive then $A_{i} \succcurlyeq 0$ for all $i \in \underline{P}$ and $a_{l g}^{(0)} \geq 0$ for $l \neq g$.

Represent (2) componentwise, i.e.,

$$
x_{l}^{\Delta}(t)=\sum_{j=1}^{n} a_{l j}^{(0)} x_{j}(t)+\sum_{i=1}^{p} \sum_{j=1}^{n} a_{l j}^{(i)} x_{j}\left(t-\tau_{i}(t)\right)-x_{l}(t),
$$

where $x_{l}(\cdot)$ is the $l$ th component of vector $x(\cdot)$. 
Proof of Lemma 2. Assume that $A_{i} \succcurlyeq 0$ for all $i \in \underline{P}_{0}, a_{j j}^{(0)} \geq 1$ for all $j \in \underline{N}$, and the initial condition $\varphi(\cdot) \succcurlyeq 0$. Then, we have $x_{l}^{\Delta}(0) \geq 0$ by (3) and therefore, $x_{l}(t) \geq 0$ for $t \in[-\tau, \sigma(0)] \cap \mathbb{T}_{0}$.

Assume for contradiction that there exists $t_{1} \in \mathbb{T}_{0,+}$ such that $x_{l}\left(t_{1}\right)<0$. Thus, there exists $t_{0} \in\left[0, t_{1}\right) \cap \mathbb{T}_{0,+}$ such that $x_{j}\left(t_{0}\right) \geq 0, x_{j}\left(t_{0}-\tau_{i}\left(t_{0}\right)\right) \geq 0$ for all $j \in \underline{N}, i \in \underline{P}$, and $x_{l}^{\Delta}\left(t_{0}\right)<0$. It follows from (3) that

$$
x_{l}^{\Delta}\left(t_{0}\right)=\sum_{j=1}^{n} a_{l j}^{(0)} x_{j}\left(t_{0}\right)+\sum_{i=1}^{p} \sum_{j=1}^{n} a_{l j}^{(i)} x_{j}\left(t_{0}-\tau_{i}\left(t_{0}\right)\right)-x_{l}\left(t_{0}\right) \geq 0,
$$

which contradicts to $x_{l}^{\Delta}\left(t_{0}\right)<0$. Therefore system (2) is positive. This completes the proof.

Proof of Lemma 3. Suppose that system (2) is positive. By Definition 2, $x(t) \succcurlyeq 0$ for all $t \in \mathbb{T}_{0,+}$ if $\varphi(\cdot) \succcurlyeq 0$.

Case 1. Assume for contradiction that there exists $q \in \underline{P}$ such that $A_{q} \succcurlyeq 0$ does not hold. Thus, there exists an element $a_{l g}^{(q)}<0$. Denote $\phi\left(-\tau_{i}(0)\right)=\left[\phi_{i 1}, \phi_{i 2}, \ldots, \phi_{i n}\right]^{T}$ and $x(0)=\left[\phi_{01}, \phi_{02}, \ldots, \phi_{0 n}\right]^{T}$. Choose $\varphi_{i j}\left(i \in \underline{P}_{0}, j \in \underline{N}\right)$ such that $\phi_{q g}>0$ and $\phi_{i j}=0$ for all $i \neq q$ or $j \neq g$. Since $q \neq 0$ then $x(0)=\left[\phi_{01}, \phi_{02}, \ldots, \phi_{0 n}\right]^{T}=[0,0, \ldots, 0]^{T}$. It follows from (3) that

$$
x_{l}^{\Delta}(0)=\sum_{i=0}^{p} \sum_{j=1}^{n} a_{l j}^{(i)} \varphi_{i j}=a_{l g}^{(q)} \varphi_{q g}<0 .
$$

Since $x_{l}(0)=0$ and $x_{l}^{\Delta}(0)<0$, then there exists $t_{0} \in \mathbb{T}_{0,+}$ such that $x_{l}\left(t_{0}\right)<0$, which contradicts the assumption that $x(t) \succcurlyeq 0$ for all $t \in \mathbb{T}_{0,+}$.

Case 2. Assume for contradiction that $a_{l g}^{(0)}<0$ for $l \neq g$. Let $\phi\left(-\tau_{i}(0)\right)=[0,0, \ldots, 0]^{T}$ and $x(0)=\left[0, \ldots, 0, \phi_{0 g}, 0, \ldots, 0\right]^{T}$ where $\phi_{0 g}>0$. It follows from (3) that

$$
x_{l}^{\Delta}(0)=\sum_{i=0}^{p} \sum_{j=1}^{n} a_{l j}^{(i)} \varphi_{i j}=a_{l g}^{(0)} \varphi_{0 g}<0 .
$$

Since $x_{l}(0)=0$ and $x_{l}^{\Delta}(0)<0$, then there exists $t_{0} \in \mathbb{T}_{0,+}$ such that $x_{l}\left(t_{0}\right)<0$, which contradicts the assumption that $x(t) \succcurlyeq 0$ for all $t \in \mathbb{T}_{0,+}$. Therefore, $A_{i} \succcurlyeq 0$ for all $i \in \underline{P}$ and $a_{l g}^{(0)} \geq 0$ for $l \neq g$. This completes the proof.

Lemma 4. Assume that system (2) is positive. Let $x_{a}(t)$ and $x_{b}(t)$ be the trajectories solutions of (2) under the initial conditions $\phi_{a}(\cdot)$ and $\phi_{b}(\cdot)$, respectively. Then $\varphi_{a}(\cdot) \preccurlyeq \varphi_{b}(\cdot)$ implies that $x_{a}(t) \preccurlyeq x_{b}(t)$ for all $t \in \mathbb{T}_{0,+}$.

Proof. It is easy to see that $x(t)=x_{b}(t)-x_{a}(t)$ is the solution to system (2) under the initial condition $\varphi(\cdot)=\varphi_{b}(\cdot)-\varphi_{a}(\cdot) \succcurlyeq 0$. Since (2) is positive, $x(t) \succcurlyeq 0$ for all $t \in \mathbb{T}_{0,+\cdot}$ This completes the proof.

\section{Main results}

The purpose of this section is to establish asymptotic stability criteria for the positive system with bounded time-varying delays on time scales. Consider the following 
positive system

$$
\begin{aligned}
& x^{\Delta}(t)=\left(A_{0}-I\right) x(t)+\sum_{i=1}^{p} A_{i} x\left(t-\tau_{i}(t)\right), \quad t \in \mathbb{T}_{0,+} \\
& x(t)=\varphi(t) \succcurlyeq 0, \quad t \in[-\tau, 0] \cap \mathbb{T}_{0},
\end{aligned}
$$

where $A_{i} \in \mathbb{R}_{0,+}^{n \times n}$ for $i \in \underline{P}, a_{l g}^{(0)} \geq 0$ for $l \neq g, t-\tau_{i}(t) \in \mathbb{T}_{0}$ for $t \in \mathbb{T}_{0,+}$, and the delays $\tau_{i}(t) \in \mathbb{T}_{0,+}$ satisfy

$$
0 \leq \alpha_{i} \leq \tau_{i}(t) \leq \tau_{i,} \quad t \in \mathbb{T}_{0,+}
$$

with constants $\alpha_{i} \in \mathbb{T}_{0,+}, \tau_{i} \in \mathbb{T}_{0,+}$, and

$$
\tau=\max \left\{\tau_{i} \mid i=1,2, \ldots, p\right\}
$$

$\varphi:[-\tau, 0] \cap \mathbb{T}_{0} \rightarrow \mathbb{R}_{0,+}^{n}$ is the vector-valued initial function. By Lemma 2, system (4) is positive.

Now, we consider the following system closely related to (4)

$$
\begin{aligned}
& y^{\Delta}(t)=\left(A_{0}-I\right) \gamma(t)+\sum_{i=1}^{p} A_{i} \gamma\left(t-\tau_{i}\right), \quad t \in \mathbb{T}_{0,+} \\
& y(t)=\phi(t) \succcurlyeq 0, \quad t \in[-\tau, 0] \cap \mathbb{T}_{0},
\end{aligned}
$$

where all the system matrices are as in (4), and $\tau_{i}$ in (7) is the supremum of $\tau_{i}(t)$ in (4), as shown in (5), $\tau$ is defined by (6), and $\phi:[-\tau, 0] \cap \mathbb{T}_{0} \rightarrow \mathbb{R}_{0,+}^{n}$ is an arbitrary initial condition. Note that the delays in (7) are constant.

Lemma 5. Consider system (7). Suppose that there exists a vector $\lambda \in \mathbb{R}_{+}^{n}$ satisfying

$$
\left(\sum_{i=0}^{p} A_{i}-I\right) \lambda \prec 0
$$

and that the initial condition is $\varphi(\cdot) \equiv \lambda$. Then, for any $t \in\left([-\tau, 0] \cap \mathbb{T}_{0}\right) \cup \mathbb{T}_{0^{+},}$, the solution to system (7) satisfies

$$
y^{\Delta}(t) \preccurlyeq 0 .
$$

Proof. Note that the initial condition $\varphi(\cdot) \equiv \lambda$. By (7), it yields that

1

$$
\gamma^{\Delta}(0)=\left(A_{0}-I\right) \gamma(0)+\sum_{i=1}^{p} A_{i} y\left(-\tau_{i}\right)=\left(\sum_{i=0}^{p} A_{i}-I\right) \lambda .
$$

It follows from $y(t)=\varphi(t) \equiv \lambda$ for all $t \in[-\tau, 0] \cap \mathbb{T}_{0}$, (8), and (10) that $\gamma^{\Delta}(t) \preccurlyeq 0$ for any $t \in[-\tau, 0] \cap \mathbb{T}_{0}$.

Now assume for contradiction that there exists $t_{0} \in \mathbb{T}_{0,+}$ such that $y^{\Delta}\left(t_{0}\right) \varphi 0$ and $\gamma^{\Delta}(t) \preccurlyeq 0$ for any $t \in\left[-\tau, t_{0}\right] \cap \mathbb{T}_{0}$. By Lemma $1, y(t)$ is right-decreasing for all $t \in\left[-\tau, t_{0}\right] \cap \mathbb{T}_{0}$. 
It follows from (7), (8), and $y(t)=\varphi(t) \equiv \lambda$ for $t \in\left[-\tau, t_{0}\right] \cap \mathbb{T}_{0}$ that

$$
\begin{aligned}
y^{\Delta}\left(t_{0}\right) & =\left(A_{0}-I\right) \gamma\left(t_{0}\right)+\sum_{i=1}^{p} A_{i} y\left(t_{0}-\tau_{i}\right) \\
& \preccurlyeq\left(A_{0}-I\right) y(0)+\sum_{i=1}^{p} A_{i} y(-\tau) \\
& \preccurlyeq\left(A_{0}-I\right) \lambda+\sum_{i=1}^{p} A_{i} \lambda \\
& =\left(\sum_{i=0}^{p} A_{i}-I\right) \lambda \\
& \prec 0,
\end{aligned}
$$

which contradicts the assumption that $y^{\Delta}\left(t_{0}\right) \varphi 0$. Therefore, $y^{\Delta}(t) \preccurlyeq 0$ for any $t \in\left([-\tau, 0] \cap \mathbb{T}_{0}\right) \cup \mathbb{T}_{0,+}$. This completes the proof.

The next lemma will show that, under certain conditions, the solution to system (4) is not greater than that to system (7), as stated in the following discussion.

Lemma 6. Suppose that there exists a vector $\lambda \in \mathbb{R}_{+}^{n}$ satisfying (8) and the initial conditions for system (4) and (7) are the same, i.e., $\phi(\cdot) \equiv \varphi(\cdot) \equiv \lambda$. Then

$$
x(t) \preccurlyeq y(t)
$$

for all $t \in \mathbb{T}_{0_{1}+}$, where $x(t)$ and $y(t)$ are solutions to (4) and (7), respectively.

Proof. By (4) and (7), it follows that

$$
x^{\Delta}(0)=\left(A_{0}-I\right) x(0)+\sum_{i=1}^{p} A_{i} x\left(-\tau_{i}(0)\right)=\left(\sum_{i=0}^{p} A_{i}-I\right) \lambda
$$

and

$$
\gamma^{\Delta}(0)=\left(A_{0}-I\right) \gamma(0)+\sum_{i=1}^{p} A_{i} y\left(-\tau_{i}\right)=\left(\sum_{i=0}^{p} A_{i}-I\right) \lambda .
$$

Hence $x^{\Delta}(0)=y^{\Delta}(0)$, which implies $x(\sigma(0))=y(\sigma(0))$.

Moreover, there exists $t_{0} \in \mathbb{T}_{0,+}$ such that $x(t)=y(t)$ for all $t \in\left[-\tau, t_{0}\right] \cap \mathbb{T}_{0}$. By Lemma 5 and (5), we have $x^{\Delta}\left(t_{0}\right) \preccurlyeq 0, \gamma^{\Delta}\left(t_{0}\right) \preccurlyeq 0$, and

$$
\begin{aligned}
x^{\Delta}\left(t_{0}\right) & =\left(A_{0}-I\right) x\left(t_{0}\right)+\sum_{i=1}^{p} A_{i} x\left(t_{0}-\tau_{i}\left(t_{0}\right)\right) \\
& \preccurlyeq\left(A_{0}-I\right) x\left(t_{0}\right)+\sum_{i=1}^{p} A_{i} x\left(t_{0}-\tau_{i}\right) \\
& =\left(A_{0}-I\right) \gamma\left(t_{0}\right)+\sum_{i=1}^{p} A_{i} \gamma\left(t_{0}-\tau_{i}\right) \\
& =\gamma^{\Delta}\left(t_{0}\right),
\end{aligned}
$$

which implies $x\left(\sigma\left(t_{0}\right)\right) \preccurlyeq y\left(\sigma\left(t_{0}\right)\right)$. In the following, we can prove that $x(t) \preccurlyeq y(t)$ for all $t \in\left(t_{0},+\infty\right) \cap \mathbb{T}_{0,+}$. 
Assume for contradiction that there exists $t_{2} \in\left(t_{0},+\infty\right) \cap \mathbb{T}_{0,+}$ such that $x\left(t_{2}\right) \varphi y\left(t_{2}\right)$. Thus, there exists $t_{1} \in\left[t_{0}, t_{2}\right) \cap \mathbb{T}_{0,+}$ such that $x(t) \preccurlyeq y(t)$ for all $t \in\left[0, t_{1}\right] \cap \mathbb{T}_{0,+}$ and $x^{\Delta}\left(t_{1}\right) \varphi y^{\Delta}\left(t_{1}\right)$. It follows from (4), (5), (7), and Lemma 5 that

$$
\begin{aligned}
x^{\Delta}\left(t_{1}\right)-y^{\Delta}\left(t_{1}\right) & =\left(A_{0}-I\right)\left[x\left(t_{1}\right)-\gamma\left(t_{1}\right)\right]+\sum_{i=1}^{p} A_{i} x\left(t_{1}-\tau_{i}\left(t_{1}\right)\right)-\sum_{i=1}^{p} A_{i} y\left(t_{1}-\tau_{i}\right) \\
& \preccurlyeq\left(A_{0}-I\right)\left[x\left(t_{1}\right)-\gamma\left(t_{1}\right)\right]+\sum_{i=1}^{p} A_{i}\left[x\left(t_{1}-\tau_{i}\right)-\gamma\left(t_{1}-\tau_{i}\right)\right] \\
& \preccurlyeq 0,
\end{aligned}
$$

which contradicts the assumption $x^{\Delta}\left(t_{1}\right)-y^{\Delta}\left(t_{1}\right) \varphi 0$. Then (11) holds for all $t \in \mathbb{T}_{0,+}$. This completes the proof.

Consider the system (7) satisfying the assumption $y(t)=\varphi(t) \otimes 0$ for all $t \in[-\tau, 0] \cap \mathbb{T}_{0}$.

Lemma 7. Let (7) be a positive system. Then the positive system (7) is asymptotically stable if and only if there exists a vector $\lambda \in \mathbb{R}_{+}^{n}$ such that (8) holds.

Proof. Necessity: By integration, it follows from (7) that

$$
\int_{0}^{t} \gamma^{\Delta}(\theta) \Delta \theta=\left(A_{0}-I\right) \int_{0}^{t} \gamma(\theta) \Delta \theta+\sum_{i=1}^{p} A_{i} \int_{0}^{t} \gamma\left(\theta-\tau_{i}\right) \Delta \theta .
$$

Then

$$
\begin{aligned}
\gamma(t)-\gamma(0) & =\left(A_{0}-I\right) \int_{0}^{t} \gamma(\theta) \Delta \theta+\sum_{i=1}^{p} A_{i} \int_{-\tau_{i}}^{t-\tau_{i}} \gamma(\theta) \Delta \theta \\
& =\left(A_{0}-I\right) \int_{0}^{t} \gamma(\theta) \Delta \theta+\sum_{i=1}^{p} A_{i}\left[\int_{0}^{t-\tau_{i}} \gamma(\theta) \Delta \theta+\int_{-\tau_{i}}^{0} \gamma(\theta) \Delta \theta\right] \\
& =\left(A_{0}-I\right) \int_{0}^{t} \gamma(\theta) \Delta \theta+\sum_{i=1}^{p} A_{i} \int_{0}^{t-\tau_{i}} \gamma(\theta) \Delta \theta+\sum_{i=1}^{p} A_{i} \int_{-\tau_{i}}^{0} \gamma(\theta) \Delta \theta .
\end{aligned}
$$

Since the system (7) is asymptotically stable, then $y(t) \rightarrow 0$ for $t \rightarrow+\infty$. It follows from

$$
\begin{aligned}
-\gamma(0) & =\left(A_{0}-I\right) \int_{0}^{+\infty} \gamma(\theta) \Delta \theta+\sum_{i=1}^{p} A_{i} \int_{0}^{+\infty} \gamma(\theta) \Delta \theta+\sum_{i=1}^{p} A_{i} \int_{-\tau_{i}}^{0} \gamma(\theta) \Delta \theta \\
& =\left(\sum_{i=0}^{p} A_{i}-I\right) \int_{0}^{+\infty} \gamma(\theta) \Delta \theta+\sum_{i=1}^{p} A_{i} \int_{-\tau_{i}}^{0} \gamma(\theta) \Delta \theta
\end{aligned}
$$

that

$$
-\gamma(0)-\sum_{i=1}^{p} A_{i} \int_{-\tau_{i}}^{0} \gamma(\theta) \Delta \theta=\left(\sum_{i=0}^{p} A_{i}-I\right) \int_{0}^{+\infty} \gamma(\theta) \Delta \theta .
$$

From assumption that $y(t) \otimes 0$ for all $t \in[-\tau, 0] \cap \mathbb{T}_{0}$ and (7) is a positive system, we have the left side of (12) is negative and 


$$
\left(\sum_{i=0}^{p} A_{i}-I\right) \int_{0}^{+\infty} \gamma(\theta) \Delta \theta \prec 0 .
$$

The condition (13) is equivalent to $\left(\Sigma_{i=0}^{p} A_{i}-I\right) \lambda \prec 0$ for $\lambda=\int_{0}^{+\infty} \gamma(\theta) \Delta \theta$.

Sufficiency: Let us consider the dual system

$$
x^{\Delta}(t)=\left(A_{0}-I\right)^{T} x(t)+\sum_{i=1}^{p} A_{i}^{T} x\left(t-\tau_{i}\right), \quad t \in \mathbb{T}_{0,+},
$$

which is positive and asymptotically stable.

As a Lyapunov function for the dual system (14) we may choose the following function

$$
V[x(t)]=x^{T}(t) \lambda+\sum_{i=1}^{p} \int_{-\tau_{i}}^{0} x^{T}(t+\theta) A_{i} \lambda \Delta \theta,
$$

which is positive for non-zero $x(t) \in \mathbb{R}_{0,+}^{n}$ and for positive $\lambda \in \mathbb{R}_{+}^{n}$.

From (14) and (15) we have

$$
\begin{aligned}
\{V[x(t)]\}^{\Delta} & =x^{\Delta^{T}}(t) \lambda+\sum_{i=1}^{p}\left[x^{T}(t)-x^{T}\left(t-\tau_{i}\right)\right] A_{i} \lambda \\
& =x^{T}(t)\left(A_{0}-I\right) \lambda+\sum_{i=1}^{p} x^{T}\left(t-\tau_{i}\right) A_{i} \lambda+\sum_{i=1}^{p}\left[x^{T}(t)-x^{T}\left(t-\tau_{i}\right)\right] A_{i} \lambda \\
& =x^{T}(t)\left(\sum_{i=0}^{p} A_{i}-I\right) \lambda .
\end{aligned}
$$

Hence, the condition $\left(\Sigma_{i=0}^{p} A_{i}-I\right) \lambda \prec 0$ implies $\{V[x(t)]\}^{\Delta} \prec 0$ and the positive system (7) is asymptotically stable. This completes the proof.

Theorem 1. Let (4) be a positive system. Then the positive system (4) is asymptotically stable if and only if there exists a vector $\lambda \in \mathbb{R}_{+}^{n}$ such that (8) holds.

Proof. Sufficiency: Assume that there exists a vector $\lambda \in \mathbb{R}_{+}^{n}$ such that (8) holds. By Lemma 7, system (7) is asymptotically stable.

Define $\|\phi\|=\sup _{t \in[-\tau, 0] \cap \mathbb{T}_{0}}\|\phi(t)\|$. Since system (7) is asymptotically stable, for any given $\varepsilon>0$, there exists a scalar $\delta>0$, such that if $\|\varphi\|<\delta$, then the corresponding solution $y_{\varphi}(t)$ to (7) satisfies that $\left\|y_{\varphi}(t)\right\|<\varepsilon$ for all $t \in \mathbb{T}_{0,+}$ and $\lim _{t \rightarrow+\infty}\left\|y_{\varphi}(t)\right\|=0$.

In particular, choose the initial condition $\varphi(\cdot)=\alpha \lambda$, where $\alpha>0$, such that $\|\alpha \lambda\|<\delta$. Then, the corresponding solution $y_{\alpha \lambda}(t)$ must satisfy that $\left\|y_{\alpha \lambda}(t)\right\|<\varepsilon$ for all $t \in \mathbb{T}_{0,+}$ and $\lim _{t \rightarrow+\infty}\left\|y_{\alpha \lambda}(t)\right\|=0$.

For system (4), let the initial condition be $\phi(\cdot)=\alpha \lambda$. By Lemma 6, the corresponding solution $x_{\alpha \lambda}(t)$ satisfies $x_{\alpha \lambda}(t) \preccurlyeq \gamma_{\alpha \lambda}(t)$ for all $t \in \mathbb{T}_{0,+}$. In addition, by Lemma 4 , for any initial condition $\varphi(\cdot) \preccurlyeq \alpha \lambda$, the corresponding solution satisfies $x_{\varphi}(t) \preccurlyeq x_{\alpha \lambda}(t)$ for all $t \in \mathbb{T}_{0,+}$.

Therefore, for system (4), arbitrary given $\varepsilon>0$, there exists $\delta_{1}=\|\alpha \lambda\|>0$ such that $\|\phi\|<\delta_{1}$, implies $\left\|x_{\phi}(t)\right\| \leq\left\|x_{\alpha \lambda}(t)\right\| \leq\left\|y_{\alpha \lambda}(t)\right\|<\varepsilon$ for all $t \in \mathbb{T}_{0,+}$. 
Furthermore, since $\left\|x_{\phi}(t)\right\| \leq\left\|y_{\alpha \lambda}(t)\right\|$ for all $t \in \mathbb{T}_{0,+}, \lim _{t \rightarrow+\infty}\left\|y_{\alpha \lambda}(t)\right\|=0$ implies

$$
\lim _{t \rightarrow+\infty}\left\|x_{\varphi}(t)\right\|=0
$$

and therefore, system (4) is asymptotically stable.

Necessity: Suppose that system (4) is asymptotically stable for any delays satisfying (5). Particularly, let $\tau_{i}(t)=\tau_{i}$, then system (7) is necessarily asymptotically stable. It follows from Lemma 7 that (8) must hold. This completes the proof.

For the continuous-time positive systems with constant delays and discrete-time positive systems with time-varying delays, $[9,19]$ have established some necessary and sufficient stability criteria. Theorem 1 can be viewed as an extension to time scales.

Remark 1. In the continuous case: $\mu(t) \equiv 0$ (i.e., $\mathbb{T}=\mathbb{R}$ ). It follows from (1) that the positive system (4) can be written as:

$$
\begin{gathered}
x^{\prime}(t)=\left(A_{0}-I\right) x(t)+\sum_{i=1}^{p} A_{i} x\left(t-\tau_{i}(t)\right), \quad t \geq 0 \\
x(t)=\varphi(t) \succcurlyeq 0, \quad t \in[-\tau, 0],
\end{gathered}
$$

where $0 \leq \tau_{i}(t) \leq \tau_{i}, \tau=\max \left\{\tau_{i} \mid i=1,2, \ldots, p\right\}$, and $\varphi:[-\tau, 0] \rightarrow \mathbb{R}_{0,+}^{n}$ is the vectorvalued initial function. Let $A_{0}-I=A, p=1, A_{1}=A_{d}$, and $\tau_{i}(t) \equiv \tau$, then the system (16) is the same as the continuous-time positive system with constant delays in [9]. Hence, Theorem 1 is the same as that obtained by Haddad and Chellaboina [9, Theorem 3.1] (see Section 1).

Remark 2. In the discrete case: $\mu(t) \equiv 1$ (i.e., $\mathbb{T}=\mathbb{N}_{0}$ ). It also follows from (1) that the positive system (4) can be written as:

$$
\begin{aligned}
& \Delta x(t)=\left(A_{0}-I\right) x(t)+\sum_{i=1}^{p} A_{i} x\left(t-\tau_{i}(t)\right), \quad t \in \mathbb{N}_{0} \\
& x(t)=\varphi(t) \succcurlyeq 0, \quad t=-\tau, \ldots, 0,
\end{aligned}
$$

where $0 \leq \tau_{i}(t) \leq \tau_{i}, \tau=\max \left\{\tau_{i} \mid i=1,2, \ldots, p\right\}$, and $\varphi:\{-\tau, \ldots, 0\} \rightarrow \mathbb{R}_{0,+}^{n}$ is the vectorvalued initial function. By $\Delta x(t)=x(t+1)-x(t)$, the above system can be rewritten as:

$$
\begin{aligned}
& x(t+1)=A_{0} x(t)+\sum_{i=1}^{p} A_{i} x\left(t-\tau_{i}(t)\right), \quad t \in \mathbb{N}_{0} \\
& x(t)=\varphi(t) \succcurlyeq 0, \quad t=-\tau, \ldots, 0 .
\end{aligned}
$$

It is evident that system (17) is of the same form as the discrete-time positive system with time-varying delays in [19]. Hence, Theorem 1 in this special case is the same as [19, Theorem 1] (see Section 1).

Remark 3. Since system (4) includes delay-independent case (i.e., $\left.\tau_{i}(t) \equiv 0\right)$, if positive system (4) is asymptotically stable, then the matrix $\left(\sum_{i=0}^{p} A_{i}-I\right)$ in (8) is a Hurwitz matrix.

Example 1. The positive systems play a key role in understanding many processes in biological science. To illustrate this, a delay-independent model of glycolysis in yeast has been constructed. The glycolysis is the name of a cellular process in which glucose is converted into different carbon compounds in a number of reaction steps. This process plays an important role in the metabolism of most organisms, and its function is 
partly to produce energy rich molecules and partly to produce carbon compounds that can be used for biosynthesis. The glycolysis has been extensively explored in many organisms and several mathematical models of it have been made. In 2000, a model of glycolysis has been made by Teusink et al. [20]. In order to make this model suitable for our system, a few modifications have been done. Such as changing state variables to constants and changing certain constants into state variables. After these changes the model contains 13 state variables, $x_{1}, \ldots, x_{13}$, each representing the concentration of a carbon compound, except from one, $x_{5}$, which represents a module of two interconvertable carbon compounds. Since the biological process is nonlinear, the system was linearized around its steady state.

$$
x^{\prime}(t)=A x(t), \quad x\left(t_{0}\right)=x_{0},
$$

where $x=\left(x_{1}, \ldots, x_{13}\right)^{T}$ is the vector of the 13 state variables and

$$
A=\left(\begin{array}{lllllllllllll}
-472.8 & 1.416 & 0 & 0 & 0 & 0 & 0 & 0 & 0 & 0 & 0 & 0 & 0 \\
408.3 & -90.04 & 487.4 & 0 & 0 & 0 & 0 & 0 & 0 & 0 & 0 & 0 & 0 \\
0 & 88.62 & -1127 & 51.52 & 0 & 0 & 0 & 0 & 0 & 0 & 0 & 0 & 0 \\
0 & 0 & 639.9 & -595.3 & 308.5 & 0 & 0 & 0 & 0 & 0 & 0 & 6.238 & 0 \\
0 & 0 & 0 & 487.5 & -787.3 & 3.361 \times 10^{4} & 0 & 0 & 0 & 0 & 0 & 0 & 0 \\
0 & 0 & 0 & 0 & 161.4 & -1.212 \times 10^{6} & 875.3 & 0 & 0 & 0 & 0 & 0 & 0 \\
0 & 0 & 0 & 0 & 0 & 1.179 \times 10^{6} & -1947 & 6881 & 0 & 0 & 0 & 0 & 0 \\
0 & 0 & 0 & 0 & 0 & 0 & 1072 & -9408 & 722 & 0 & 0 & 0 & 0 \\
0 & 0 & 0 & 0 & 0 & 0 & 0 & 2527 & -2137 & 3.938 & 0 & 0 & 0 \\
0 & 0 & 0 & 0 & 0 & 0 & 0 & 0 & 1415 & -10.25 & 0 & 0 & 0 \\
0 & 0 & 0 & 0 & 0 & 0 & 0 & 0 & 0 & 6.582 & -2480 & 0 & 7.645 \\
0 & 0 & 0 & 0 & 8.984 & 0 & 0 & 0 & 0 & 0 & 0 & -126.2 & 0 \\
0 & 0 & 0 & 0 & 0 & 0 & 0 & 0 & 0 & 0 & 2438 & 0 & -10.24
\end{array}\right)
$$

Since $A$ is a Hurwitz matrix and all its eigenvalues lie in the left-half of the complex plane, see Table 1 , the positive system (18) is asymptotically stable. Let

$$
\lambda=(0.8573,0.9775,0.8683,0.6662,0.7332,0.8488,0.8446,0.8550,0.8802,0.8561,0.7579,0.7304,0.5982)^{T} \succ 0
$$

then we have

$$
\begin{aligned}
A \lambda & =10^{3} \times(-0.0062,-0.0099,-0.0758,-0.0611,-0.2282,-8.3188,-0.0077,-0.0079,-0.0523, \\
& -0.0003,-0.4212,-0.0876,-0.0003)^{T} \prec 0 .
\end{aligned}
$$

By Theorem 1, we can also deduce that (18) is an asymptotically stable positive system.

Table 1 Eigenvalues of matrix $A$

\begin{tabular}{c}
$A$ \\
\hline$-1.212856733058425 \times 10^{6}$ \\
$-0.010420961874116 \times 10^{6}$ \\
$-0.002487523768668 \times 10^{6}$ \\
$-0.002022791184840 \times 10^{6}$ \\
$-0.001280501517495 \times 10^{6}$ \\
$-0.001040020002847 \times 10^{6}$ \\
$-0.000474117073543 \times 10^{6}$ \\
$-0.000341099069420 \times 10^{6}$ \\
$-0.000213407895628 \times 10^{6}$ \\
$-0.000126041203210 \times 10^{6}$ \\
$-0.000045765211863 \times 10^{6}$ \\
$-0.000002716231332 \times 10^{6}$ \\
$-0.000006451908612 \times 10^{6}$
\end{tabular}




\section{Acknowledgements}

Many thanks to Leonid Berezansky (the editor) and the anonymous reviewers for helpful comments and suggestions. This research was supported by the NNSF of China (Grant Nos. 11071143, 11101241, and 61174217), the NNSF of Shandong Province (Grant Nos. ZR2009AL003, ZR2010AL002, ZR2010AL016, ZR2011AL007, and JQ201119), the Scientific Research and Development Project of Shandong Provincial Education Department (J11LA01), and the NSF of University of Jinan (XKY0918).

\section{Authors' contributions}

YS directed the study and helped inspection. CZ carried out the the main results of this article, established the model, and drafted the manuscript. All the authors read and approved the final manuscript.

\section{Competing interests}

The authors declare that they have no competing interests.

Received: 30 January 2012 Accepted: 9 May 2012 Published: 9 May 2012

\section{References}

1. Berman, A, Neumann, M, Stern, RJ: Nonnegative Matrices in Dynamic Systems. New York, Wiley (1989)

2. Kaczorek, T: Positive 1D and 2D Systems. Springer-Verlag, London (2002)

3. Kaczorek, T: Polynomial and Rational Matrices, Applications in Dynamical Systems Theory. Springer-Verlag, London (2007)

4. Gorecki, H: Analysis and Synthesis of Control Systems with Delay. WNT, Warszawa (1971)

5. Gorecki, H, Fuksa, S, Grabowski, P, Korytowski, A: Analysis and Synthesis of Time Delay Systems. PWN-J. Wiley, WarszawaChichester (1989)

6. Gorecki, H, Korytowski, A: Advances in Optimization and Stability Analysis of Dynamical Systems. Publishing Department of University of Mining and Metallurgy, Krakow (1993)

7. Buslowicz, M: Robust Stability of Dynamical Linear Stationary Systems with Delays. Publishing Department of Technical University of Bialystok, Warszawa-Bialystok (2000)

8. Niculescu, SI: Delay E ects on Stability. A Robust Control Approach. Springer-Verlag, London (2001)

9. Haddad, WM, Chellaboina, V: Stability theory for nonnegative and compartmental dynamical systems with time delay. Syst Control Lett. 51, 355-361 (2004). doi:10.1016/j.sysconle.2003.09.006

10. Hinrichsen, D, Hgoc, PHA, Son, NK: Stability radii of positive higher order difference systems. Syst Control Lett. 49, 377-388 (2003). doi:10.1016/50167-6911(03)00116-6

11. Buslowicz, M, Kaczorek, T: Stability and robust stability of positive linear discrete-time systems with pure delay. In Proc 10th IEEE Int Conf on Methods and Models in Automation and Robotics, Miedzyzdroje, vol. 1, pp. 105-108.IEEE, Piscataway (2004)

12. Buslowicz, M: Stability of positive singular discrete-time systems with unit delay with canonical forms of state matrices. In Proc 12th IEEE Int Conf on Methods and Models in Automation and Robotics, Miedzyzdroje, vol. 2006, pp. 215-218. IEEE, Piscataway (2006)

13. Buslowicz, M: Robust stability of positive discrete-time linear systems with multiple delays with linear unity rank uncertainty structure or nonnegative perturbation matrices. Bull Pol Acad Sci Tech Sci. 55, 1-5 (2007)

14. Rami, MA, Tadeo, F, Benzaouia, A: Control of constrained positive discrete systems. In Proc Am Control Conf, New York, vol. 2007, pp. 5851-5856.IEEE, Piscataway (2007)

15. Rami, MA, Tadeo, F: Positive observation problem for linear discrete positive systems. In Proc 45th IEEE Conf Decision Control, San Diego, vol. 2006, pp. 4729-4733.IEEE, Piscataway (2006)

16. Hmammed, A, Benzaouia, A, Rami, MA, Tadeo, F: Positive stabilization of discrete-time systems with unknown delay and bounded controls. In Proc Eur Control Conf, Kos, vol. 2007, pp. 5616-5622.European Union Control Association, Zurich (2007)

17. Buslowicz, M: Simple stability conditions for linear positive discrete-time systems with delays. Bull Pol Acad Sci Tech Sci. 56, 325-328 (2008)

18. Yu, W, Zhong, S: Constrained control of positive discrete-time systems with delays. IEEE Trans Circ Syst II Exp Briefs. 55, 193-197 (2008)

19. Liu, X, Yu, W, Wang, L: Stability analysis of positive systems with bounded time-varying delays. IEEE Trans Circ Syst II Exp Briefs. 56, 600-604 (2009)

20. Teusink, B, Passerge, J, Reijenga, CA, Esgalhado, E, van der Weijden, CC, Schepper, M, Walsh, MC, Bakker, BM, van Dam, K, Westerhoff, HV, Snoep, JL: Can yeast glycolysis be understood in terms of in vitro kinetics of the constituent enzymes? testing biochemistry. Eur J Biochem. 267, 5313-5329 (2000). doi:10.1046/j.1432-1327.2000.01527.x

doi:10.1186/1687-1847-2012-56

Cite this article as: Zhang and Sun: Stability analysis of linear positive systems with time delays on time scales. Advances in Difference Equations 2012 2012:56. 